\title{
Luminescence and scintillation properties of Mg-
}

\section{codoped LuAG:Pr single crystals annealed in air}

\author{
Jan Pejchal $^{1 *}$, Maksym Buryi ${ }^{1}$, Vladimir Babin ${ }^{1}$, Petr Prusa ${ }^{1,2}$, Alena Beitlerova ${ }^{1}$, Jan Barta ${ }^{1,2}$, Lubomir Havlak ${ }^{1}$, \\ Kei Kamada ${ }^{3,4}$, Akira Yoshikawa ${ }^{3,4}$, Valentin Laguta ${ }^{1}$, Martin Nikl ${ }^{1}$ \\ ${ }^{1}$ Institute of Physics AS CR, Cukrovarnicka 10, Prague 6, 16200, Czech Republic \\ ${ }^{2}$ Faculty of Nuclear Sciences and Physical Engineering, Czech Technical University in Prague, Brehova 7, \\ Prague 1, 11519, Czech Republic \\ ${ }^{3}$ Institute for Materials Research, Tohoku University, 2-1-1, Katahira, Aoba-ku, Sendai, Miyagi, 980-8577, \\ Japan \\ ${ }^{4}$ New Industry Creation Hatchery Center, Tohoku University, 6-6-10 Aoba, Aramaki, Aoba-ku, Sendai, Miyagi \\ 980-8579, Japan
}

\section{*e-mail: pejchal@fzu.cz}

Abstract

The influence of the $\mathrm{Mg}^{2+}$ codoping and annealing in the air on the scintillation and luminescence characteristics of Pr-doped lutetium-aluminum garnet crystals (LuAG) was studied to find a possible positive effect of $\mathrm{Pr}^{4+}$. The overall scintillation efficiency under X-ray excitation of the annealed Pr- doped samples decreased with increasing Mg concentration. This was explained by increased overlap of the $\operatorname{Pr}^{3+} 5 \mathrm{~d}-4 \mathrm{f}$ emission with the charge-transfer (CT) absorption band of the $\mathrm{Pr}^{4+}$ ion stabilized by $\mathrm{Mg}^{2+}$. This absorption caused even greater decrease of the light yield, as the light is collected from the whole sample volume in the pulse-height spectrum measurement. Electron centers based on oxygen vacancies ( $\mathrm{F}^{+}$centers) which can have negative influence on scintillation mechanism were revealed by the electron paramagnetic resonance (EPR) experiments. The $\mathrm{O}^{-}$hole center was detected as well and the temperature dependences of the EPR spectra showed that its thermal stability was decreased by $\mathrm{Mg}$ codoping. Negligible changes in the decay time of $\operatorname{Pr}^{3+} 5 \mathrm{~d}-4 \mathrm{f}$ photoluminescence exclude the non-radiative energy transfer from relaxed $5 d_{1}$ excited state of $\operatorname{Pr}^{3+}$ towards CT absorption transition of $\mathrm{Pr}^{4+}$ and suggest that simple reabsorption of the emitted light takes place. Unlike for the Ce-doped analogues, promoting the $\mathrm{Pr}^{3+}$ luminescence center to the tetravalent state by divalent ion co-doping and air annealing did not improve scintillation properties of Pr-doped LuAG. 
Keywords: Scintillation; Pr4+; luminescence; codoping; lutetium-aluminum garnet

\section{Introduction}

Inorganic scintillation single crystals have been employed in the ionizing radiation detection and related applications for several decades [1]. They are utilized in high-energy physics, environmental monitoring, geological survey and oil well logging or astronomy. Medical imaging and especially security scanning are the applications mostly stimulating the development of new scintillation materials due to increasing demands on their performance.

In several modern inorganic scintillators fast $5 \mathrm{~d}-4 \mathrm{f}$ luminescence of $\mathrm{Ce}^{3+}$ has been employed to obtain the scintillation response of the order of several tens of ns with high efficiency (see [1] and references therein). Lutetium aluminum garnet $\left(\mathrm{Lu}_{3} \mathrm{Al}_{5} \mathrm{O}_{12}\right.$, $\left.\mathrm{LuAG}\right)$ single crystal matrix appears as a very suitable host for the $\mathrm{Ce}^{3+}$ and other rare earth (RE) luminescence centers due to the Lu site where the $\mathrm{RE}^{3+}$ ion can be easily substituted, high density of $6.67 \mathrm{~g} / \mathrm{cm}^{3}$, high effective atomic number and mechanical and chemical and thermal resistance [2]. The first systematic studies started almost 2 decades ago [3]. Besides favorable scintillation properties, one of the main demerits of this material was presence of slow components in the scintillation decay caused by retrapping of electrons at the traps related to $\mathrm{Lu}_{\mathrm{Al}}$ intrinsic anti-site defects (AD) [4], generally present also in some other aluminum garnets due to high crystal growth temperature [5]. This phenomenon caused serious deterioration of the light yield and timing performance. However, it is well known that the flexibility of the garnet matrix allows incorporation of other elements [6] and therefore the lattice constants, band-gap and other parameters such as depth and trapping efficiency of the AD-related traps can be easily tuned. This approach led to development of new slow-component-free and highly efficient Ce-doped scintillator based on the Gd, Gaadmixed multicomponent garnet [7]. Another recent approach to improve the scintillation performance is employing $\mathrm{Ce}^{4+}$ stabilized with divalent ion $\left(\mathrm{Mg}^{2+}, \mathrm{Ca}^{2+}\right)$ codoping in the scintillation mechanism [2, 8-10]. The $\mathrm{Ce}^{4+}$ can be regarded as $\mathrm{Ce}^{3+}$ with already captured hole, which is ready for an immediate capture of thermalized electron from the conduction band followed by forming the $\mathrm{Ce}^{3+}$ in an excited state. The stable $\mathrm{Ce}^{4+}$ center directly competes with shallow electron traps responsible for the slow decay components. The de-excitation of this temporary $\mathrm{Ce}^{3+}$ results in the usual $\mathrm{Ce}^{3+}$ emission which might show somewhat different emission characteristics regarding e.g. temperature stability $[8,11]$. Finally, a nonradiative hole capture from a nearby defect or valence band leads to the $\mathrm{Ce}^{4+}$ center regeneration $[12,13]$. This mechanism was reported to be responsible for improvement of scintillation properties of lutetium silicate crystals [8] and also LuAG:Ce optical 
ceramics, where $\mathrm{Mg}^{2+}$ was used as a sintering aid [9]. Another way to stabilize Ce in its tetravalent form in garnets is annealing in an oxidizing atmosphere [14]. The annealed crystals showed increased light yield and reduced proportion of the slow components in the scintillation decay. The suppression of the slow components is caused also by the reduction of concentration of deep traps related to oxygen vacancies.

Another suitable luminescence center is the $\operatorname{Pr}^{3+}$ ion. Some Pr-doped scintillators were briefly investigated in the past [15]. To obtain fast and high-energy-shifted 5d-4f emission of $\operatorname{Pr}^{3+}$ the crystal field of the host matrix should be strong enough to shift the lowest $5 \mathrm{~d}$-state well below the ${ }^{1} \mathrm{~S}_{0}$ one to make the $5 \mathrm{~d}-4 \mathrm{f}$ radiative transition possible. For details on these aspects of the $\operatorname{Pr}^{3+}$ luminescence, see for example [16]. High quality Pr-doped $\mathrm{Lu}_{3} \mathrm{Al}_{5} \mathrm{O}_{12}$ (LuAG:Pr) was announced as a fast and efficient scintillator [17,18] and an attempt of its application in positron emission mammography has been already made [19]. Analogous problems with the retrapping at AD’s were observed [17]. Similar attempt of composition engineering using Gd and Ga admixture failed in the case of Pr-doped multicomponent garnets due to overlap of the $\operatorname{Pr}^{3+} 5 \mathrm{~d}-4 \mathrm{f}$ emission with the $\mathrm{Gd}^{3+}{ }^{8} \mathrm{~S}_{7 / 2} \rightarrow{ }^{6} \mathrm{P}_{\mathrm{J}}$ transition and resulting energy back-transfer from the $\operatorname{Pr}^{3+}$ to the host [20]. There is a question if stabilizing $\operatorname{Pr}^{4+}$ by divalent ion codoping or annealing would improve the scintillation properties of garnet-based scintillators. Radiation hardness and some luminescence properties of Mg-codoped LuAG:Pr were studied recently and no positive influence was found mainly due to presence of $\mathrm{Yb}^{3+}$ and other impurities [21, 22]. However, systematic study of scintillation properties of Mg-codoped LuAG:Pr is still missing. Thus the aim of this work is to investigate absorption, luminescence and scintillation properties of Mg-codoped and annealed LuAG:Pr for different concentrations of Mg codopant.

\section{Materials and Experimental Methods}

The crystals were grown by micro-pulling-down method [23, 24] with radiofrequency inductive heating. An iridium crucible with a die of $3 \mathrm{~mm}$ in diameter was used. The growth was performed under $\mathrm{N}_{2}(4 \mathrm{~N})$ atmosphere using $<111>$ oriented LuAG single crystal as a seed. The pulling speed was around 0.07-0.1 $\mathrm{mm} / \mathrm{min}$. The starting materials were prepared by mixing $4 \mathrm{~N}$ purity $\mathrm{Lu}_{2} \mathrm{O}_{3}, \mathrm{Al}_{2} \mathrm{O}_{3}$ and $\mathrm{Pr}_{6} \mathrm{O}_{11}$ powders. $\mathrm{MgCO}_{3}$ of $4 \mathrm{~N}$ purity was added to the mixture for the Mg-codoped samples.

The LuAG:Pr samples with Pr concentration of 0.2 atomic \% were prepared. The Mg concentration was 0 , 300 or 3000 atomic ppm. It is reasonable to suppose that Pr substitutes for Lu while Mg substitutes for Al, even though it can be incorporated in all the three kinds of sites available within the garnet structure [6]. Therefore the Pr concentration is given with respect to $\mathrm{Lu}$, while the Mg concentration is given with respect to Al. Then 
we can specify the hypothetical formula of the grown crystal as $\mathrm{Lu}_{3(1-\mathrm{x})} \mathrm{Pr}_{3 \mathrm{x}} \mathrm{Al}_{5(1-\mathrm{y})} \mathrm{Mg}_{5 \mathrm{y}} \mathrm{O}_{12}$, where $\mathrm{x}=0.002$ and $y=0,0.0003$ and 0.003 . However, the atomic $\%$ or atomic ppm will be used for simplicity further in the text to specify the sample composition. One completely undoped crystal was also grown for comparison.

The crystal rods had diameter of $3 \mathrm{~mm}$ and were from 35 to $45 \mathrm{~mm}$ in length. $1 \mathrm{~mm}$ thick circular samples were cut from approximately $1 \mathrm{~cm}$ from the beginning (marked as START) and $1 \mathrm{~cm}$ from the end (marked as END) of the rod and mirror-polished for further optical characterization. The samples were annealed in air for $12 \mathrm{~h}$ at a temperature of $1200^{\circ} \mathrm{C}$.

The phase purity of prepared crystals was examined by XRD analysis using the Rigaku MiniFlex 600 powder X-ray diffractometer equipped with Ni-filtered $\mathrm{Cu}-\mathrm{K} \alpha_{1,2}$ line and $\mathrm{NaI}$ :Tl scintillation detector. Prior to the measurement, a selected part of the prepared crystal was grinded into a fine powder in an agate mortar and its diffraction pattern was then measured in continuous mode between $10^{\circ}$ and $80^{\circ} 2 \theta$. The diffraction patterns recorded were fully consistent with the ICDD PDF-2 record 01-73-1368 $\left(\mathrm{Lu}_{3} \mathrm{Al}_{5} \mathrm{O}_{12}\right)$ and showed some evidence of slight preferred orientation due to the grinding of a single-crystalline material.

Luminescence measurements were performed by a modified spectrofluorometer (custom made 5000M model, Horiba Jobin Yvon) equipped with TBX-04 photon counting detector (IBH Scotland). A deuterium steady-state lamp was used for the excitation and emission spectra measurements. An X-ray tube operating at a voltage of $40 \mathrm{kV}$ and $15 \mathrm{~mA}$ current with Mo anode (ISO-DEBYEFLEX 3003 Seifert Gmbh.) was used as an excitation source for the radioluminescence (RL) measurements. All the spectra were corrected for experimental distortions. The hydrogen nanosecond flashlamp or pulsed nanoLED source (IBH Scotland) were used for the luminescence decay measurements using the methods of time correlated single photon counting. Exponential fits of the decays were obtained by performing the convolution of the considered function with the instrumental response and a least-square sum fitting procedure (SpectraSolve software package from Ames Photonics Inc.). For the light yield measurements, sample crystals were optically coupled with a hybrid photomultiplier (HPMT) DEP PPO 475B. Samples were coupled to the HPMT by silicon grease and wrapped in teflon tape used as a reflector (preliminary, $\mathrm{BaSO}_{4}$ was used also for one sample, but the result was practically the same as for Teflon). The ${ }^{137} \mathrm{Cs}(661.6 \mathrm{keV})$ source was used for excitation and the amplifier shaping time was $6 \mu$ s. The scintillation decay kinetics was measured with a fast photomultiplier and Tektronix TDS3052C digital Phosphor Oscilloscope under ${ }^{137}$ Cs excitation. Scintillaton decay data were evaluated by SpectraSolve as well. All the optical and scintillation measurements were performed at room temperature (RT). 
The electron paramagnetic resonance (EPR) spectra were measured using the commercial Bruker X-/Qband E580 FT/CW ELEXSYS spectrometer at X-band (microwave frequency $9.5 \mathrm{GHz}$ ) within the temperature range 10-296 K using an Oxford Instruments ESR900 continuous flow cryostat. As a source of X-ray irradiation the same ISO-DEBYEFLEX 3003 X-Ray equipment mentioned above was used. The X-ray tube $55 \mathrm{kV}$ voltage and $30 \mathrm{~mA}$ current were kept during the samples irradiation at liquid nitrogen temperature (LNT).

\section{Results and discussion}

The absorption spectra of the Pr-doped samples are compared with the undoped one in the figure 1 . Only the START parts from each sample were chosen, as the spectra of the END parts did not show any significant differences. For the Pr-doped Mg-free sample, the curve gradually goes up above the baseline between 300-600 $\mathrm{nm}$ and one can notice prominent relatively sharp peaks related to $\operatorname{Pr}^{3+} 4 f-5 d_{1}$ and $4 f-5 d_{2}$ transitions that appear at 285 and $240 \mathrm{~nm}$, respectively. This result corresponds to the observations in the literature (see [2] and references therein). The curve sharply increases below $200 \mathrm{~nm}$ which is most probably due to defect states modifying the band edge. For the sample codoped with $300 \mathrm{ppm} \mathrm{Mg}$, the spectrum is very similar but the increase of the absorption between $300-600 \mathrm{~nm}$ is more pronounced. The absorption at the shortest wavelengths is significantly smaller. Concerning the sample codoped with $3000 \mathrm{ppm} \mathrm{Mg}$, the absorption between 300-600 $\mathrm{nm}$ is very strong and obscures the features related to $\operatorname{Pr}^{3+}$ and band edge at the shorter wavelengths. The intensity of this absorption increases with increasing $\mathrm{Mg}$ concentration and it was ascribed to the chargetransfer (CT) transition of the $\mathrm{Pr}^{4+}$ ion, similarly as the analogous $\mathrm{Ce}^{4+} \mathrm{CT}$ transition observed for Ce-doped scintillation materials codoped with divalent alkaline earth ions [8, 10-13]. As can be expected, annealing causes the oxidation of Pr to its tetravalent state while the $\mathrm{Mg}^{2+}$ codoping enhances its stability. The effect is especially prominent for the sample with $3000 \mathrm{ppm} \mathrm{Mg}$, which follows from the charge compensation of the divalent $\mathrm{Mg}^{2+}$ by $\operatorname{Pr}^{4+}$. It is not excluded that some $\operatorname{Pr}^{4+}$ ions are already present in the Mg-codoped samples before annealing. Comparative study of the properties before and after annealing is under way and will be published elsewhere, as some peculiarities in the luminescence behavior can be observed. Considering that the onset of the $\operatorname{Pr}^{4+} \mathrm{CT}$ transition is situated around $600 \mathrm{~nm}$ and that the $\mathrm{Ce}^{4+} \mathrm{CT}$ absorption for the Ce doped scintillators has an onset around $340 \mathrm{~nm}$, the difference of the onset positions corresponds to some $1.5 \mathrm{eV}$, which exactly matches the difference between $4 \mathrm{f}$ ground state energies of $\mathrm{Ce}^{3+}$ and $\operatorname{Pr}^{3+}[25]$ and provides further support for the ascription of the smooth absorption band below $600 \mathrm{~nm}$. It is worth noting that in the spectra for both Mg-doped samples that a new sharp peak at $213 \mathrm{~nm}$ emerges after annealing. Its origin is unknown but might be related to 
simultaneous occurrence of $\mathrm{Pr}^{4+}$ and $\mathrm{Mg}^{2+}$, as the $\mathrm{Pr}^{4+} \mathrm{CT}$ absorption is present also in the Mg-free sample

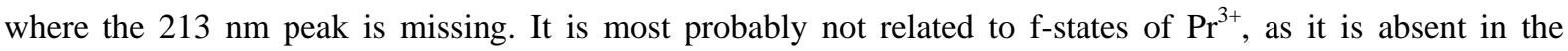
photoluminescence excitation spectra of $\mathrm{Pr}^{3+}$ emission (see below). In the undoped sample, the absorption at 250 nm may be related to the iron impurity, which can change valence from 2+ to 3+ during annealing [26].

The comparison of the RL spectra of the studied samples under X-ray excitation with that for the $\mathrm{Bi}_{4} \mathrm{Ge}_{3} \mathrm{O}_{12}$ (BGO) commercially used reference scintillator is in the figure 2. They are dominated by the $5 \mathrm{~d}-4 \mathrm{f}$ emission peak at $308 \mathrm{~nm}\left(5 \mathrm{~d}-{ }^{3} \mathrm{H}_{4}\right)$ and a shoulder around $360 \mathrm{~nm}$ caused by transitions from the $5 \mathrm{~d}$ state to the higher $4 \mathrm{f}$ levels $\left({ }^{3} \mathrm{H}_{5,6},{ }^{3} \mathrm{~F}_{3,4}\right)$. The shoulder at the short wavelength side of the $308 \mathrm{~nm}$ peak originates from host luminescence related to recombination of exciton at AD's. It is worth noting that its intensity is practically the same for both the Mg concentrations. Very weak $4 \mathrm{f}-4 \mathrm{f}$ transitions can be observed at longer wavelengths. The overall scintillation efficiency of all the samples is significantly higher than that of BGO scintillator and is slightly lower for the Mg-codoped samples. The cause can be an overlap of the $\operatorname{Pr}^{3+} 5 \mathrm{~d}-4 \mathrm{f}$ emission peak with the broad absorption between 300-600 nm discussed above, which increases with increasing Mg concentration. Interestingly, the RL intensity for both Mg-codoped samples is practically the same. All the results shown here are again for the START parts. Only minimum differences were observed for the samples from the END parts.

The photoluminescence (PL) excitation spectrum of the $308 \mathrm{~nm}$ emission is shown in the figure 3a for the 300 ppm Mg Pr-doped LuAG sample (START). The dominant peaks at 280 and $235 \mathrm{~nm}$ corresponding to the 4f-5d transitions are already observed in the absorption spectra in the figure 1.

We guess that the slight shift to shorter wavelengths might be caused by an instrumental effect, as practically no changes can be observed for the $4 \mathrm{f}-5 \mathrm{~d}$ peaks in the absorption spectra in the figure 1 . The small peak below $200 \mathrm{~nm}$ is related to the defect states shaping the band edge. The $213 \mathrm{~nm}$ peak discussed above was not found at all, which again supports the idea that it is not related to $\operatorname{Pr}^{3+}$ ion. The same is valid for the excitation spectra of the other samples.

The comparison of the photoluminescence emission spectra for the same sample LuAG:Pr 0.2\% Mg 300 ppm (START) under $200 \mathrm{~nm}$ and $285 \mathrm{~nm}$ excitation is in the figure 3b. Both spectra are again dominated by the $\mathrm{Pr}^{3+}$ 5d-4f luminescence and their shape is also comparable with the radioluminescence one (figure 2), but the host emission shoulder below $300 \mathrm{~nm}$ is obviously missing. For the spectrum excited under $200 \mathrm{~nm}$ (from valence band top to the defect levels near the bottom edge of the conduction band), the $\mathrm{Pr}^{3+} 4 \mathrm{f}-4 \mathrm{f}$ emission is quite apparent while it is almost missing in the spectrum excited under $285 \mathrm{~nm}$. The pronounced $4 \mathrm{f}-4 \mathrm{f}$ emission can 
be related to the simultaneous excitation of the $\operatorname{Pr}^{3+}$ ion and some defect state from which an energy leak to the other f-levels takes place. The assignment of the $4 \mathrm{f}-4 \mathrm{f}$ lines in the figure $3 \mathrm{~b}$ was made according to [27].

It is worth mentioning that the shoulder related to the transitions from the $5 \mathrm{~d}$ level to the higher $4 \mathrm{f}$ ones at $360 \mathrm{~nm}$ changes its relative intensity with respect to the dominant $5 \mathrm{~d}-4 \mathrm{f}$ peak at $308 \mathrm{~nm}$. It does not show any stable trend with the $\mathrm{Mg}$ concentration. Interestingly, the absorption of the $\mathrm{F}^{+}$center based on an oxygen vacancy in LuAG lies exactly in this spectral area [28]. Its creation might be modified by Mg codoping and annealing and it can influence the PL intensity in this region. Presence of an electron-like center (most probably the $\mathrm{F}^{+}$one) was proved by the EPR measurements below.

Another emission band emerged at $249 \mathrm{~nm}$ upon $200 \mathrm{~nm}$ excitation in the emission spectrum (figure 3b). This band can be related to an emission of some defect induced by annealing and stabilized by $\mathrm{Mg}^{2+}$ codopant. We should emphasize that this wavelength exactly corresponds with the emission of the low-temperature configuration of the AD- trapped exciton (TE) reported in [29]. It might be that this low-temperature trapped exciton configuration is stabilized by Mg co-doping. Note that the shape of this band might be further obscured by $\mathrm{Pr}^{3+} 4 \mathrm{f}-5 \mathrm{~d}$ absorption bands at 245 and $280 \mathrm{~nm}$. Due to this overlap an energy transfer from the defect levels to the $5 \mathrm{~d}$ ones can take place, which can have an influence on the scintillation decay kinetics. As mentioned above, the intensity of the $\operatorname{Pr}^{3+} 4 \mathrm{f}-4 \mathrm{f}$ emission peaks in this spectrum are much more pronounced with respect to the spectrum under direct excitation at $285 \mathrm{~nm}$. It seems that the levels related to the defect emitting at $249 \mathrm{~nm}$ can be responsible for the population of the $\operatorname{Pr}^{3+} 4 \mathrm{f}$-levels. This effect is slightly more apparent also for the sample codoped with 3000 ppm Mg (not shown here).

The photoluminescence decay kinetics for the LuAG:Pr 0.2\% Pr (START) under direct excitation to the $4 \mathrm{f}-$ $5 \mathrm{~d}_{1}$ transition is in the figure 4 and is governed by a decay component with 21 ns decay time belonging to the 5d-4f $\mathrm{Pr}^{3+}$ emission. On the other hand, it seems that some slow component with a decay time around $200 \mathrm{~ns}$ emerges. Similar feature was observed for the other samples again. Concerning both of the Mg-codoped samples, the decay curve looked very similar and the leading decay time was shortened to around $19 \mathrm{~ns}$. The decay curves under $200 \mathrm{~nm}$ excitation were very similar and followed the trends described for the direct excitation.

The observed very slight acceleration of the photoluminescence decay kinetics with increasing $\mathrm{Mg}$ concentration can be caused by a weak energy transfer from the excited $\operatorname{Pr}^{3+} 5 \mathrm{~d}_{1}$ state towards the $\mathrm{Pr}^{4+} \mathrm{CT}$ state, as there is the spectral overlap of the $5 \mathrm{~d}-4 \mathrm{f}$ emission with the CT absorption, see above. Similar effect was observed for the samples from the END part. 
The emission spectrum under excitation into the unknown absorption sharp band at $213 \mathrm{~nm}$ discussed above is in the figure 5 . It consists of the $5 \mathrm{~d}-4 \mathrm{f}$ bands around $310 \mathrm{~nm}$ and the $4 \mathrm{f}-4 \mathrm{f}$ bands at longer wavelength, which are much more pronounced in this case. The ${ }^{3} \mathrm{P}_{0^{-}}{ }^{3} \mathrm{H}_{4}$ transition at $488 \mathrm{~nm}$ is quite apparent and was not observed in the previously discussed emission spectra. It is interesting to note that even though the $5 \mathrm{~d}-4 \mathrm{f}$ emission is excited at $213 \mathrm{~nm}$, it is not found in the excitation spectrum of this emission. The $213 \mathrm{~nm}$ wavelength exactly corresponds with the position of the ${ }^{1} \mathrm{~S}_{0}$ state of $\mathrm{Pr}^{3+}$. If this peak is ascribed to the transition to this level, it should be seen in the excitation spectra as well, as one could expect a nonradiative energy transfer from this level to the $5 d$ one. However, this is not true (figure 3a). The decay kinetics of the $\operatorname{Pr}^{3+} 5 d-4 f$ emission under 213 nm excitation is very similar as that in the figure 4, just the leading decay time is the shortest observed in all the experiments with the value of 17 ns (not shown here). Further research is necessary to clarify the origin of the $213 \mathrm{~nm}$ peak.

The scintillation light yield was determined from the pulse-height spectra under $667 \mathrm{keV}$ gamma ray excitation from ${ }^{137} \mathrm{Cs}$ source. The example of such a spectrum is in the figure 6 , where the fitted curve for the LuAG:Pr 0.2\% END sample is displayed. The full-absorption peak is clearly observed, but it interferes with Lu escape peak at slightly lower energy. However, peaks are separated enough and escape peak area is approximately 6 times smaller than area of full-absorption peak. Therefore, the measurement precision is not affected significantly by Lu escape peak.

The light yield values for all the samples including START and END parts are summarized in the Figure 7 (the $0 \mathrm{ppm}$ concentration is indicated as $1 \mathrm{ppm}$ to allow setting the logarithmic axis). A substantial decrease of the light yield with Mg codoping is observed for both parts of the Mg-codoped crystals. This decrease is the same for both START and END parts and seems to be independent on the Mg concentration similarly as radioluminescence intensities in the figure 2. On the other hand, the magnitude of the decrease of the light yield values for the samples with $\mathrm{Mg} 300 \mathrm{ppm}$ and $3000 \mathrm{ppm}$ with respect to the Mg-free one does not correspond to the decrease of the steady-state radioluminescence intensities (figure 2). This may be caused by much shorter penetration depth of X-ray excitation photons and reflection geometry used in RL measurement compared to allthe-volume excitation by $662 \mathrm{keV}$ gamma photons and transmission geometry used in LY measurement. Slow components introduced by Mg-codoping or annealing through which the light is emitted outside the $6 \mu$ s shaping time window leading to the decrease of the light yield cannot be eventually excluded as well.

Decay kinetics under the gamma-ray excitation for the LuAG:Pr0.2\% Mg 0 ppm and $\mathrm{Mg} 3000 \mathrm{ppm}$ (START) is shown in the figures 8a and 8b. Fitting the curves with the sum of exponentials was complicated 
and often 4 or 5 components were necessary, which may be a consequence of the fact that the scintillation decay of the selected samples does not follow the usual exponential decay law. In the log-log scale (see figure 8) the course of the slower component following the fast exponential part is close to a straight line, which suggests that this part follows the inverse power law. Therefore the curves were fit with a sum of an exponential component and a power function according to the equation $\mathrm{I}=\mathrm{A} \exp (-\mathrm{t} / \mathrm{tau})+\mathrm{a}(\mathrm{b}+\mathrm{ct})^{-\mathrm{p}}$, where $\mathrm{A}, \mathrm{a}, \mathrm{b}, \mathrm{c}$ and $\mathrm{p}$ are constants, tau stands for the decay constant and $t$ is time. The inverse power decay course was described in the past [31] and observed in the Ce-doped LuAG scintillators [32]. It was ascribed to the tunneling of the electrons from the AD-related shallow traps towards adjacent $\mathrm{Ce}^{3+}$ with a trapped hole (which is $\mathrm{Ce}^{4+}$ ). The usual values of the parameter p range between 0.9-1.5. For more details about tunneling-related luminescence processes see for example [30] and also references therein. It is reasonable to assume that the same phenomenon takes place in the Pr-doped analogues.

In the curves for both the Mg-free and the Mg $3000 \mathrm{ppm}$ samples the fast exponential component has a decay time of $21.6 \mathrm{~ns}$ and $21 \mathrm{~ns}$ respectively and is ascribed to the $5 \mathrm{~d}-4 \mathrm{f}$ luminescence of the $\mathrm{Pr}^{3+}$ center in the crystals. The almost negligible shortening can be again related to the energy transfer from the $\operatorname{Pr}^{3+}$ excited $5 \mathrm{~d}$ state to the $\mathrm{Pr}^{4+} \mathrm{CT}$ one. The slower component described with the power function is due to the mentioned tunneling of the electrons to from the AD-related traps and the parameter p has values of 0.73 and 0.82 for the $\mathrm{Mg}$-free and $\mathrm{Mg}$ $3000 \mathrm{ppm}$ sample respectively. These values ale slightly out of the range specified above, but it might be that there are other exponential components of small amplitude and substantially long decay time that may obscure the observed power decay kinetics. Taking into account that the value of p determines the slope of the power function in the log-log scale, it means that for the Mg-codoped sample the scintillation response is accelerated at the longer times when compared to the Mg-free sample.

Summarizing the influence of Mg codoping and annealing on luminescence and scintillation properties, we can state that it enhances the formation and stability of $\mathrm{Pr}^{4+}$ ion. This effect increases with increasing $\mathrm{Mg}$ concentration. The $\operatorname{Pr}^{4+}$ CT band is responsible for reabsorption of the $5 d-4 f$ emission coming from $\operatorname{Pr}^{3+}$ ions. The $\mathrm{Pr}^{3+}$ ions can be either regular ones or the temporary ones created in an alternative scintillation mechanism, which would be analogous with the mechanism taking part in LuAG:Ce codoped with divalent ions and which is described in the introduction and treated in $[2,8,10,11]$. The nonradiative energy transfer from $5 d_{1}$ relaxed excited state of $\operatorname{Pr}^{3+}$ towards CT state of $\operatorname{Pr}^{4+}$ is probably responsible for very slight photoluminescence (and also scintillation) decay shortening. However, as this process is almost negligible, a simple reabsorption of the $5 \mathrm{~d}-4 \mathrm{f}$ emission by the CT transition is supposed to be the dominant process which leads to decrease of RL intensity 
and scintillation light yield for the Mg-codoped samples. Further studies are needed to explain the observed independence of this effect on Mg concentration. The decrease of the RL intensity upon Mg codoping is much smaller than the decrease of the scintillation light yield. It can be tentatively explained by different excitation energies and experiment geometries as mentioned above. Moreover, an introduction of very slow components by Mg-codoping, through which most of the scintillation light is emitted outside the corresponding time window in the light yield measurement cannot be excluded as well.

The selected samples were studied by EPR before and after X-ray irradiation at approximately $77 \mathrm{~K}$. The samples were LuAG:Pr0.2\% START and END, LuAG:Pr 0.2\%, Mg 300 ppm START and END, and LuAG:Pr 0.2\%, Mg 3000 ppm START and END. The EPR spectra measured at 40K prior to an X-ray irradiation contained mostly resonance lines coming from $\mathrm{Yb}^{3+}$ ions, see for example the spectrum for the LuAG:Pr 0.2\% START in the figure 9a. The $\mathrm{Yb}^{3+}$ was already described in LuAG [33] and in our samples it is obviously present as an accidental impurity. Moreover, there is also a number of lines of unknown origin (Figure 9a) which could not be properly identified due to their low intensities. No $\mathrm{Pr}^{4+}$ resonance lines were found in the spectra at all. The $\operatorname{Pr}^{4+}$ spectral lines would be easy identified due to pronounced hyperfine structure produced by ${ }^{141} \operatorname{Pr}$ nucleus having $100 \%$ natural abundance and the nuclear spin $5 / 2$. Therefore, either their position is out of the EPR spectrometer magnetic field range or the concentration of these ions is too low to be detectable. When the samples were exposed to the X-ray irradiation at liquid nitrogen temperature, new lines emerged in the spectra in the 3300-3450 G magnetic field range (see Figure 9b,c), near $g$ factor 2.

The resonance line marked as 1 in Figure 9b,c was ascribed to the g-factor value $g \approx 2.026$ whereas the spectral position of the line marked as 2 is characterized by the $g \approx 1.949$. The g-factor value for the first one is common for the hole-like centers, namely $\mathrm{O}^{-}$defects [34-40], since it is a bit higher than 2.0023, which is the value for a free electron. Remarkably, the position of the line 1 is almost independent on the orientation of the external magnetic field with respect to the $<100>$ crystallographic direction. The $g$ factor of the paramagnetic center responsible for this line is thus almost isotropic. In principle, the $\mathrm{O}^{-}$defects show very small or no anisotropy of the $g$ factor, especially in cubic lattices $[13,34-40]$. The line 1 could be then ascribed to the $\mathrm{O}^{-}$ defect. The presence of $\mathrm{O}^{-}$defect further supports the existence of the alternative scintillation mechanism similar to the Mg-codoped LuAG:Ce [2, 10, 11]. It involves the $\mathrm{O}^{-}$center which releases a hole to regenerate the tetravalent luminescence ion in the final stage of the mechanism.

The line 2, which is observed only in the crystal with the highest Mg concentration, shows the isotropy of the $g$ factor as well. Most probably, it belongs to the electron captured at an oxygen vacancy forming an $\mathrm{F}^{+}$ 
center, since such centers similarly to the $\mathrm{O}^{-}$ones show no or weak anisotropy of the $g$ factor, see for example $[34,41]$. The intensity of this line does not change after heating of a sample up to RT and holding on at this temperature for 1 day (see Figure 9b). Prompt heating of the sample up to approximately 420-450 K makes no effect as well. However, after keeping the sample for four days at RT, the center completely disappeared. $\mathrm{F}^{+}$ centers can be very stable [40,41] surviving annealing even at the temperatures much higher than RT [40]. Moreover, it is possible that even after annealing in air not all the oxygen vacancies and related defects were removed in the Pr $0.2 \% \mathrm{Mg} 3000 \mathrm{ppm}$ (START) sample. It might be that the local lack of charge due to divalent $\mathrm{Mg}^{2+}$ codopant embedded at a trivalent site can stabilize the oxygen vacancies. Such an effect is expected to be significant especially in the samples with high $\mathrm{Mg}$ concentration, which is the case of this studied sample. However, such defects responsible for the line 2 were not detected in the END counterpart. Taking into account small or no differences in the luminescence and scintillation characteristics of both parts, such an observation is hard to explain so far and further study is necessary to clarify this discrepancy.

Due to similarity of the spectra measured in Pr0.2\% START and END samples, the spectrum measured for the END sample was not shown in Figure 9c.

It was found from the temperature dependences of the EPR signal that the $\mathrm{O}^{-}$defects in the Pr only doped samples and those the Pr,Mg co-doped have different thermal stability, which was observed from measurements of the dependence of the intensity of the line 1 (Figure $9 \mathrm{~b}, \mathrm{c}$ ) on temperature for the LuAG:Pr0.2\% START and END samples together with the Pr0.2\% Mg 300 ppm START and Pr0.2\% Mg 3000 ppm START. The corresponding dependences are shown in the figure 10 together with the fitted curves.

In this experiment the spectra were measured always at the reference temperature of $40 \mathrm{~K}$ but the sample was quickly heated up to the chosen temperature $T_{h}$ and then promptly cooled down to the reference temperature, each time increasing $\mathrm{T}_{\mathrm{h}}$ with the step of $10-15 \mathrm{~K}$.

The Pr 0.2\% START and END samples showed roughly the same temperature dependences of the $\mathrm{O}^{-}$ EPR intensity (Figure 10). With Mg codoping the stability of the $\mathrm{O}^{-}$center decreased and seems again almost independent on the $\mathrm{Mg}$ concentration as the dependences for the $300 \mathrm{ppm}$ or $3000 \mathrm{ppm} \mathrm{Mg}^{2+}$ sample are quite similar especially at the highest temperatures.

The dependences shown in figure 10 have been fitted with the calculated curves according to the expressions 1 and 2 presented below. Each of them describes the hole trap kinetics (first order - Eq. 1, and second order - Eq. 2, respectively) which were adapted for the aims of the above described heating method mentioned in [42]. 


$$
\begin{aligned}
& I_{i+1}=I_{i} \exp \left(-f_{01} t \exp \left[-E_{t 1} / k_{B} T_{i}\right]\right), i=0,1, \ldots, \mathrm{m}, \\
& I_{i+1}=\frac{I_{i}}{1+\frac{I_{i} f_{02}}{P} \exp \left(-E_{t 2} / k_{B} T_{i}\right)}, i=0,1, \ldots, \mathrm{m},
\end{aligned}
$$

Here the EPR intensity $I_{i}$ [43], is proportional to the trapped holes concentration remaining after each heating cycle $i ; P$ is the coefficient which depends on the whole amount of traps in the crystal, and transition coefficients [42]. $E_{t 1}$ and $E_{t 2}, f_{01}$ and $f_{02}$ are the trap depths and frequency factors for the first or second order kinetics, respectively. The parameters determined from the fit are: $E_{t 1}=0.338 \pm 0.005 \mathrm{eV}, f_{01}=(1 \pm 0.5) \times 10^{8} \mathrm{~s}^{-}$ ${ }^{1} ; E_{t 2}=0.105 \pm 0.005 \mathrm{eV}, f_{02}=(1 \pm 0.5) \times 10^{1} \mathrm{~s}^{-1}$.

The dependence in the Pr-only doped samples shows a very unusual course since it does not obey either of the kinetics separately but the combination of them. However, the second-order kinetics (Eq. 2) prevails. The $f_{02}$ value obtained from fit does not have much physical sense since the frequency factor is mostly found within $10^{6}-10^{12} \mathrm{~s}^{-1}$ range [36, 41, 44, 45]. The calculated trap depth $E_{t 2}$ is very big and considering the $\mathrm{O}^{-}$to be a shallow trap, most probably we deal with some kind of thermally-assisted tunneling described for the Ce-doped analogue in [30].

As mentioned above, the Mg codoping significantly decreases the stability of the centers and the kinetics becomes the first order one (Eq. 1) with the usual values of the trap depth and frequency factor.

When compared with the other observations [34-36, 45], the relatively high thermal stability of the holes (see Figure 10) in all the samples suggests that the holes must be stabilized by some imperfection nearby which can be further modified by Mg codoping. Correlated thermoluminescence experiments would bring deeper insight in the above described processes, but they will be published in the forthcoming more detailed comparative study of both the annealed and as-grown samples.

\section{Conclusions}

This study focuses on the influence of Mg codoping and annealing on luminescence and scintillation properties. Both Mg codoping and annealing lead to promotion of Pr to its tetravalent state and moreover the Mg codopant enhances its stability, which increases with increasing $\mathrm{Mg}$ concentration. $\mathrm{The} \operatorname{Pr}^{4+} \mathrm{CT}$ band is 
responsible for absorption of the 5d-4f emission coming from $\mathrm{Pr}^{3+}$ ions. A very slight photoluminescence (and also scintillation) decay acceleration may be caused by an almost negligible nonradiative energy transfer from the $\operatorname{Pr}^{3+} 5 d_{1}$ level to the $\operatorname{Pr}^{4+}$ CT state. As this process is not significant, it means that the absorption by the CT state is responsible for the decrease of RL intensity and scintillation light yield for the Mg-codoped samples. Further studies are needed to explain the observed independence of this effect on Mg concentration. The decrease of the RL intensity upon Mg codoping is much smaller than the decrease of the scintillation light yield which can be tentatively explained by different excitation energies and geometries of radioluminescence and light yield measurements. This would imply that the absorption by the $\operatorname{Pr}^{4+} \mathrm{CT}$ state might be hampered in thin films and thus positive effects of divalent ion codoping can be expected for Pr-doped garnet scintillators, which have faster response when compared to the Ce-doped counterparts.

The scintillation decay curves are composed of a fast component related to $\operatorname{Pr}^{3+}$ emission and a slower component described by a power function, which indicates a tunneling recombination process from the traps related to the anti-site defects. This was already observed in the Ce-doped analogues and therefore such an interpretation is plausible.

The EPR spectra measurements revealed presence of $\mathrm{O}^{-}$centers in all the samples, which further supports the existence of the alternative scintillation mechanism in the Mg-codoped crystals. It involves the $\mathrm{O}^{-}$center which releases a hole to regenerate the tetravalent luminescence ion in the final stage of the mechanism. The temperature dependence of the EPR spectra showed that Mg codoping significantly decreases the stability of this center. The EPR experiments revealed also presence of centers based on oxygen vacancies, most probably an $\mathrm{F}^{+}$centers, which are stable above room temperature. They were observed only in the crystal with the highest Mg concentration which would mean that in this case $\mathrm{Mg}$ on a trivalent site stabilizes the oxygen vacancies due to charge compensation and makes them resistant to annealing in air.

\section{Acknowledgments}

This work has been partially supported by

(i) Czech Science Foundation (GACR) 15-18300Y project 
(ii) Development of Systems and Technology for Advanced Measurement and Analysis (SENTAN), Japan Science and Technology Agency (JST),

(iii) Health Labour Sciences Research Grant, The Ministry of Health Labour and Welfare,

(iv) The New Energy and Industrial Technology Development Organization (NEDO),

(v) Supporting Industry program, Ministry of Economy, Trade and Industry (METI) and

(vi) Association for the Progress of New Chemical Technology.

(vii) Japan Society for the Promotion of Science (JSPS)

(vii) Adaptable and Seamless Technology Transfer Program through Target-driven R\&D (A-STEP), JST,

(ix) Ministry of Education, Youth and Sports of Czech Republic, projects SAFMAT LM2015088 and LO1409

In addition, we would like to thank following persons for their support: Tohoku University, and Mr. Hiroshi Uemura, Ms. Keiko Toguchi, Ms. Megumi Sasaki, Ms. Yuka Takeda and Ms. Kuniko Kawaguchi of IMR, Tohoku University.

References

1. M. Nikl, A. Yoshikawa, Recent R\&D trends in inorganic single crystal scintillator materials for radiation detection. Adv. Opt. Mater. 3 (2015) 463-481

2. M. Nikl, A. Yoshikawa, K. Kamada, K. Nejezchleb, C. R. Stanek, J. A. Mares, K. Blazek, Development of LuAG-based scintillator crystals- A review, Progress in Crystal Growth and Characterization of Materials 59 (2013) 47-72

3. M. Nikl, E. Mihokova, J.A. Mares, A. Vedda, M. Martini, K. Nejezchleb, K. Blazek, Traps and Timing Characteristics of LuAG:Ce ${ }^{3+}$ scintillator, Phys. Status Solidi (b) 181 (2000) R10-R12

4. M. Nikl, J. A. Mares, N. Solovieva, J. Hybler, A. Voloshinovskii, K. Nejezchleb, K. Blazek, Energy transfer to the $\mathrm{Ce}^{3+}$ centers in $\mathrm{Lu}_{3} \mathrm{Al}_{5} \mathrm{O}_{12}$ scintillator, Physica Status Solidi A 201 (2004) R41 - R44 
5. V. Lupei, A. Lupei, C. Tiseanu, S. Georgescu, C. Stoicescu, P. M. Nanau, High-resolution optical spectroscopy of YAG:Nd; A test for structural and distribution models, Physical Review B 51 (1995) 8-17

6. D. Mateika, E. Volkel, J. Haisma, lattice-constant-adaptable crystallographics. II. Czochralski growth from multicomponent melts of homogeneous mixed-garnet crystals, J. Cryst. Growth 102 (1990) 994-1013

7. K. Kamada, T. Yanagida, T. Endo, K. Tsutumi, Y. Fujimoto, A. Fukabori, A. Yoshikawa, J. Pejchal, M. Nikl, Composition engineering in Ce doped $(\mathrm{Lu}, \mathrm{Gd})_{3}(\mathrm{Ga}, \mathrm{Al})_{5} \mathrm{O}_{12}$ single crystal scintillators, Cryst. Growth Des. 11 (2011) $4484-4490$

8. S. Blahuta , A. Bessiere , B. Viana , P. Dorenbos , V. Ouspenski, Evidence and Consequences of Ce in LYSO: Ce, Ca and LYSO: Ce, Mg Single Crystals for Medical Imaging Applications, IEEE. Trans. Nucl. Sci. 60 (2013) 3134-3141

9. S. Liu, X. Feng, Z. Zhou, M. Nikl, Y. Shi, Y. Pan, Effect of $\mathrm{Mg}^{2+}$ co-doping on the scintillation performance of LuAG:Ce ceramics, Phys. Status Solidi RRL 8 (2014) 105-109

10. M. Nikl, K. Kamada, V. Babin, J. Pejchal, K. Pilarova, E. Mihokova, A. Beitlerova, K. Bartosiewicz, S. Kurosawa, and A. Yoshikawa, Defect Engineering in Ce-Doped Aluminum Garnet Single Crystal Scintillators, Cryst. Growth Des. 14 (2014) 4827 - 4833

11. Y. Wu, F. Meng, Q. Li, M, Koschan, C. L. Melcher, Role of $\mathrm{Ce}^{4+}$ in the Scintillation Mechanism of Codoped $\mathrm{Gd}_{3} \mathrm{Ga}_{3} \mathrm{Al}_{2} \mathrm{O}_{12}$ :Ce, Physical Review Applied 2 (2014) 044009 (1-13)

12. Ch. Hu, Sh. Liu, M. Fasoli, A. Vedda, M. Nikl, Xi. Feng, Yu. Pan, O- centers in LuAG:Ce,Mg ceramics, Phys. Status Solidi RRL 9 (2015) 245-249

13. M. Nikl, V. Babin, J. Pejchal, V. V. Laguta, M. Buryi, J. A. Mares, K. Kamada, S. Kurosawa, A. Yoshikawa, D. Panek, P. Bruza, K. Mann, M. Müller, The stable $\mathrm{Ce}^{4+}$ center: a new tool to optimize Ce-doped oxide scintillators. IEEE Trans. Nucl. Sci. 63 (2016) 433-438 
14. M. Nikl, V. Babin, J. A. Mares, K. Kamada, S. Kurosawa, A. Yoshikawa, J. Tous, J. Houzvicka, K. Blazek, The role of cerium variable charge state in the luminescence and scintillation mechanism in complex oxide scintillators: The effect of air annealing, Journal of Luminescence 169 (2016) 539-543

15. C. W. E. van Eijk, P. Dorenbos, R. Visser, $\mathrm{Nd}^{3+}$ and $\mathrm{Pr}^{3+}$ doped inorganic scintillators, IEEE Trans. Nucl. Sci. 41, 738 (1994)

16. A. M. Srivastava, Aspects of $\operatorname{Pr}^{3+}$ luminescence in solids, J. Lumin. 169 (2016) 445-449

17. M. Nikl, H. Ogino, A. Krasnikov, A. Beitlerova, A. Yoshikawa, T. Fukuda, Photo- and radioluminescence of Pr-doped $\mathrm{Lu}_{3} \mathrm{Al}_{5} \mathrm{O}_{12}$ single crystal, Phys. Stat. Sol. A 202 (2005) R4-R6

18. H. Ogino, A. Yoshikawa, M. Nikl, K. Kamada, T. Fukuda, Scintillation characteristics of Pr-doped $\mathrm{Lu}_{3} \mathrm{Al}_{5} \mathrm{O}_{12}$ single crystals, J. Cryst. Growth 292 (2006) 239-242

19. A. Yoshikawa, T. Yanagida, K. Kamada, Y. Yokota, J. Pejchal, A. Yamaji, Y. Usuki, S. Yamamoto, M. Miyake, K. Kumagai, K. Sasaki, T.R. dos Santos, M. Baba, M. Ito, M. Takeda, N. Ohuchi, M. Nikl, Positron emission mammography using Pr:LuAG scintillator - Fusion of optical material study and systems engineering. Opt. Mater. 32 (2010) 1294 - 1297.

20. V. Babin, M. Nikl, K. Kamada, A. Beitlerova, A. Yoshikawa, Effect of the $\mathrm{Pr}^{3+} \rightarrow \mathrm{Gd}^{3+}$ energy transfer in multicomponent garnet single crystal scintillators, J. Phys. D: Appl. Phys. 46 (2013) 365303 (1-6)

21. M.V. Derdzyan, K.L. Ovanesyan, A.G. Petrosyan, A. Belsky, C. Dujardin, C. Pedrini, E. Auffray, P. Lecoq, M. Lucchini, K. Pauwels, Radiation hardness of LuAG:Ce and LuAG:Pr scintillator crystals, Journal of Crystal Growth 361 (2012) 212-216

22. A.G. Petrosyan, K.L. Ovanesyan, M.V. Derdzyan, I. Ghambaryan, G. Patton, F. Moretti, E. Auffray, P. Lecoq, M. Lucchini, K. Pauwels, C. Dujardin, A study of radiation effects on LuAG:Ce(Pr) co-activated with Ca, Journal of Crystal Growth 430 (2015) 46-51 
23. A. Yoshikawa, M. Nikl, G. Boulon, T. Fukuda, Challenge and study for developing of novel single crystalline optical materials using micro-pulling-down method, Opt. Mater. 30 (2007) 6-10

24. A. Yoshikawa, V. I. Chani, Growth of Optical Crystals by the Micro-Pulling-Down Method, Mat. Res. Soc. Bull. 34 (2009) 266-270

24. P. Dorenbos, Systematic behaviour in trivalent lanthanide charge transfer energies, J. Phys.: Condens. Matter 15 (2003) 8417-8434

26. C. Y. Chen, G. J. Pogatshnik, Y. Chen, M. R. Kokta, Optical and electron paramagnetic resonance studies of Fe impurities in yttrium aluminum garnet crystals, Phys. Rev. B 38 (1988) 8555-8561

27. A. Strzęp, W. Ryba-Romanowski, R. Lisiecki, P. Solarz, X. Xu, J. Di, J. Xu, Spectroscopic peculiarities of praseodymium impurities in $\mathrm{Lu}_{3} \mathrm{Al}_{5} \mathrm{O}_{12}$ single crystal, J. Alloys Compd. 550 (2013) 173-178

28. V. Babin, V.V. Laguta, A. Maaroos, A. Makhov, M. Nikl, S. Zazubovich, Luminescence of F ${ }^{+}$-type centers in undoped $\mathrm{Lu}_{3} \mathrm{Al}_{5} \mathrm{O}_{12}$ single crystals, Phys. Status Solidi B 248 (2011) 239-242

29. V. Babin, K. Blazek, A. Krasnikov, K. Nejezchleb, M. Nikl, T. Savikhina, S. Zazubovich, Luminescence of undoped LuAG and YAG crystals, Phys. stat. sol. (c) 2 (2005) 97-100

30. M. Nikl, A. Vedda, M. Fasoli, I. Fontana, V. V. Laguta, E. Mihokova, J. Pejchal, J. Rosa, K. Nejezchleb, Shallow traps and radiative recombination processes in $\mathrm{Lu}_{3} \mathrm{Al}_{5} \mathrm{O}_{12}$ :Ce single crystal scintillators, Physical Review B 76 (2007) 195121(1-8)

31. M. Tachyia and A. Mozumder, Kinetics of geminate-ion recombination by electron tunneling, Chem. Phys. Lett. 34 (1975) 77-79

32. D. J. Huntley, An explanation of the power-law decay of luminescence, J. Phys.: Condens. Matter 18 (2006) 1359-1365 
33. V.V. Laguta, A.M. Slipenyuk, M.D. Glinchuk, M. Nikl, J. Rosa, A. Vedda, K. Nejezchleb, Paramagnetic impurity defects in LuAG and LuAG: Sc single crystals, Opt. Mater. 30 (2007) 79-81

34. V.V. Laguta, M. Buryi, J. Rosa, D. Savchenko, J. Hybler, M. Nikl, S. Zazubovich, T. Kärner, C. R. Stanek, and K. J. McClellan, Electron and hole traps in yttrium and lutetium orthosilicate scintillator crystals: the critical role of Si-unbound oxygen, Phys. Rev. B 90 (2014) 064104 (1-12)

35. M. Buryi, D.A. Spassky, J. Hybler, V. V. Laguta, and M. Nikl, Electron Spin Resonance study of charge trapping in $\alpha-\mathrm{ZnMoO}_{4}$ single crystal scintillator, Opt. Mat. 47 (2015) 244-250

36. M. Buryi, P. Bohacek, K. Chernenko, A. Krasnikov, V.V. Laguta, E. Mihokova, M. Nikl, S. Zazubovich, Phys. Status Solidi B, Luminescence and photo-thermally stimulated defect-creation processes in $\mathrm{Bi}^{3+}$-doped single crystals of lead tungstate, 1-16 (2016) / DOI 10.1002/pssb.201552697

37. A. Watterich, L. Kovács, R. Würz, F. Schön, A. Hofstaetter, A. Scharmann, Electron spin-resonance (ESR) and electron-nuclear double-resonance (ENDOR) study of the self-trapped hole in ZnWO4 single crystals, J. Phys.: Condens. Matter 13 (2001) 1595-1607

38. S. Lenjer, O.F. Schirmer, and H. Hesse, The structure of the paramagnetic lithium center in zinc oxide and berrylium oxide, Phys. Rev. B 66 (2002) 165106 (1-12)

39. V.V. Laguta, M. Buryi, M. Nikl, J. Rosa, S. Zazubovich, Hole capture in $\mathrm{PbWO}_{4}: \mathrm{Mo}, \mathrm{La}(\mathrm{Y})$ scintillator crystals, Phys. Rev. B 83 (2011) 094123 (1-5)

40. D. Pan, G. Xu, L. Lu, Y. Yong, X. Wang, J. Wan, G. Wang, Observation and manipulation of paramagnetic oxygen vacancies in Co-doped $\mathrm{TiO}_{2}$ nanocrystals, Appl. Phys. Lett. 89 (2006) 082510(1-3).

41. W. Gellerman, F. Lüty, K. P. Koch, H. Welling, Tuneable laser operation of $\mathrm{F}_{2}^{+}$and $\left(\mathrm{F}_{2}^{+}\right)_{\mathrm{A}}$ centers in $\mathrm{OH}^{-}$ and $\mathrm{SH}^{-}$doped alkali halides, Opt. Commun. 35 (1980) 430-434

42. S. W. S. Mc Keever, Thermoluminescence of solids, Cambridge University Press (1985)

43. A. Abragam, B. Bleaney, Electron paramagnetic resonance of transition ions. vol. 1 Clarendon Press, Oxford (1970) 560-568

44. G. F. J. Garlick, A. F. Gibson, The Electron Trap Mechanism of Luminescence in Sulphide and Silicate Phosphors, Proc. Phys. Soc. 60 (1948) 574-590

45. V.V. Laguta, M. Nikl, A. Vedda, E. Mihokova, J. Rosa, K. Blazek, Hole and electron traps in the $\mathrm{YAlO}_{3}$ single crystal scintillator, Phys. Rev. B 80 (2009) 045114 (1-10) 
List of figure captions

Figure 1. Absorption spectra of the START parts of the annealed LuAG:Pr0.2\% samples with 0, 300 and 3000 ppm of Mg codopant

Figure 2. Comparison of radioluminescence spectra of the START parts of the annealed LuAG:Pr0.2\% samples with 0,300 and $3000 \mathrm{ppm}$ of $\mathrm{Mg}$ codopant

Figure 3a. photoluminescence excitation spectrum for the $308 \mathrm{~nm}$ emission for the LuAG:Pr $0.2 \% 300 \mathrm{ppm} \mathrm{Mg}$ sample

Figure 3b. Comparison of the photoluminescence spectra of the annealed Mg $300 \mathrm{ppm}$ LuAG:Pr 0.2\% sample (START) under $285 \mathrm{~nm}$ (direct 4f-5d excitation ) and $200 \mathrm{~nm}$ (to the defect states below conduction band)

Figure 4. Photoluminescence decay kinetics of the $\mathrm{Pr}^{3+} 5 \mathrm{~d}-4 \mathrm{f}$ emission at $308 \mathrm{~nm}$ under $285 \mathrm{~nm}$ excitation (direct 4f-5d) for the annealed Mg-free LuAG:Pr 0.2\% sample (START)

Figure 5. Photoluminescence spectra of the annealed Mg 300 ppm LuAG:Pr 0.2\% sample (START) under 213 nm excitation

Figure 6. Example fo the pulse-height spectrum for the LuAG:pr 0.2\% END part under $662 \mathrm{keV}$ gamma-ray excitation $\left({ }^{137} \mathrm{Cs}\right)$

Figure 7. Dependence of the light yield for the annealed LuAG:Pr0.2\% samples on concentration of $\mathrm{Mg}$ codopant.

Figure 8. Scintillation decay kinetics under gamma-ray excitation for the annealed LuAG:Pr0.2\% START (a) and LuAG:Pr0.2\% Mg 3000 ppm START (b)

Figure 9. EPR spectra measured at the orientation of the external magnetic field parallel with the $\langle 100\rangle$ axis for non-irradiated LuAG:Pr 0.2\% START (a), X-ray irradiated LuAG:Pr 0.2\%, Mg 3000 ppm START (b), LuAG:Pr $0.2 \%$ START, Pro.2\% Mg 300 ppm START and END, Pr0.2\% Mg 3000 ppm END after X-ray irradiation. 1 and 2 mark the resonance lines coming from the hole- and electron-like centers, respectively.

Figure 10. Dependence of the $\mathrm{O}^{-}$line EPR intensity on heating temperature $\mathrm{T}_{\mathrm{h}}$ measured for the Pr0.2\% START and END samples and Pr0.2\% Mg $300 \mathrm{ppm}$ START and Pr0.2\% Mg 3000 ppm START samples at fixed temperature $\mathrm{T}=40 \mathrm{~K}$. Solid lines are the calculated dependences. 
Fig 1

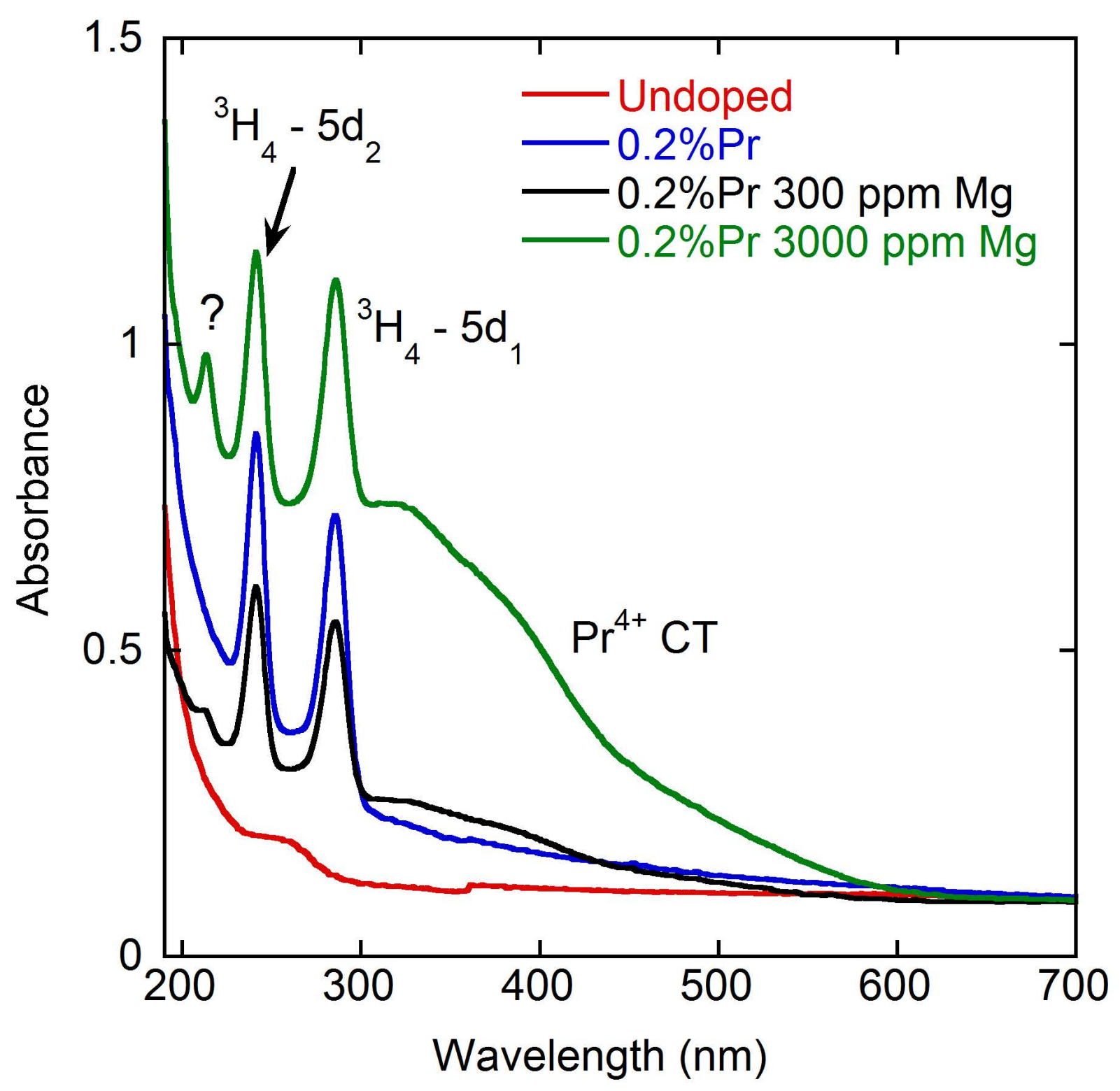


Fig 2

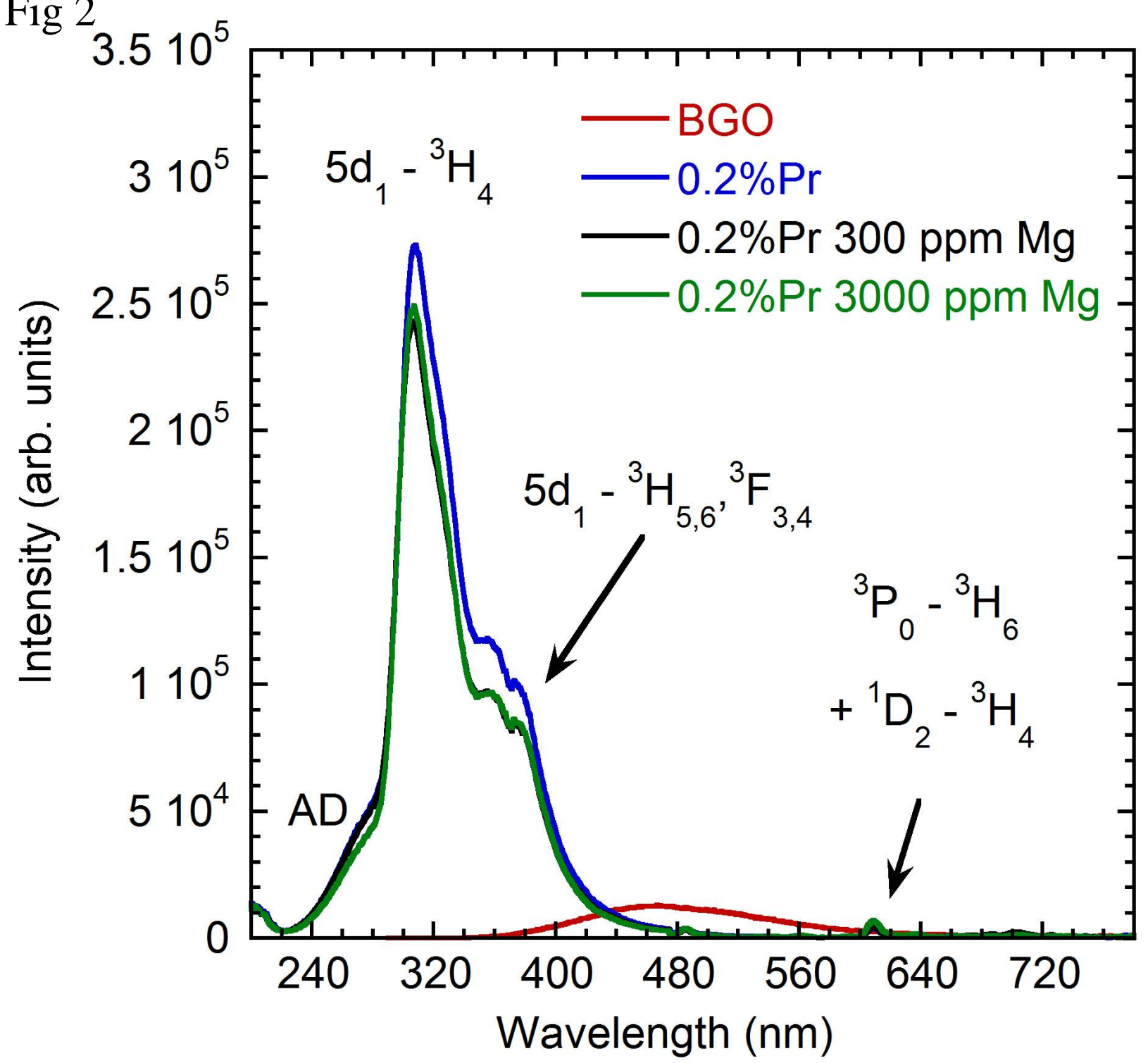


Fig $3 \mathrm{a}$

LuAG:Pr $0.2 \%$, Mg 300ppm START

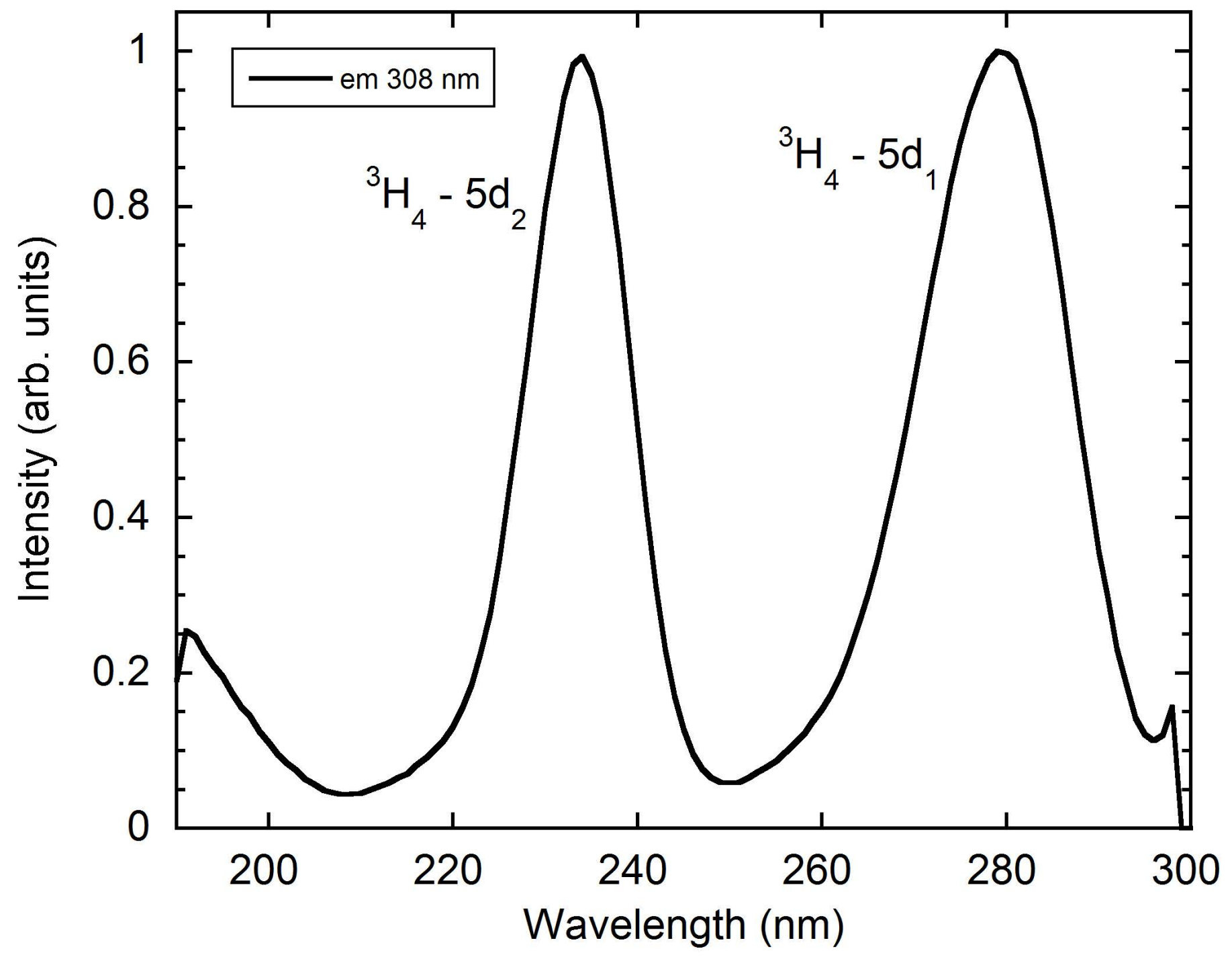


Fig 3b

LuAG:Pr $0.2 \%$, Mg 300ppm START

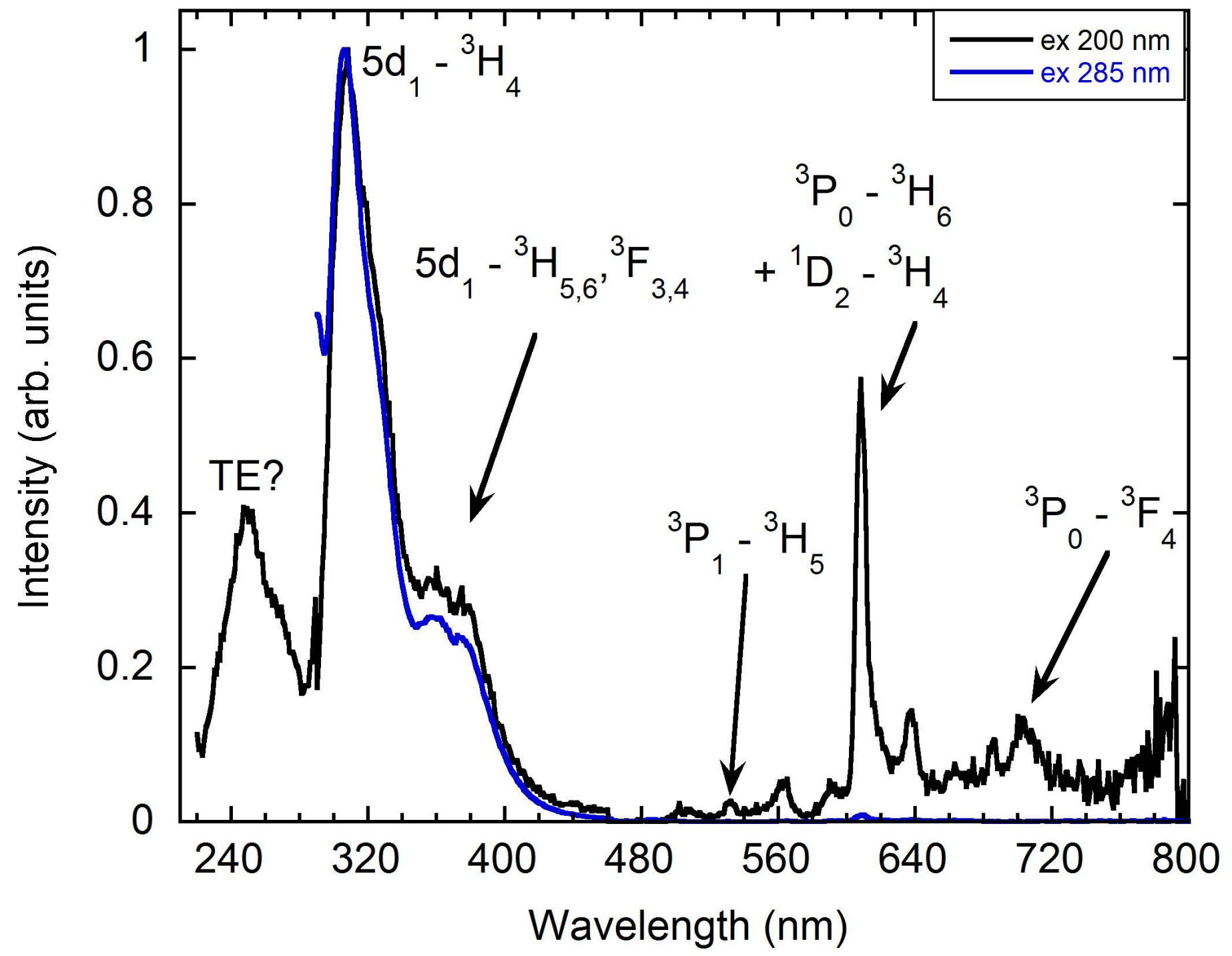




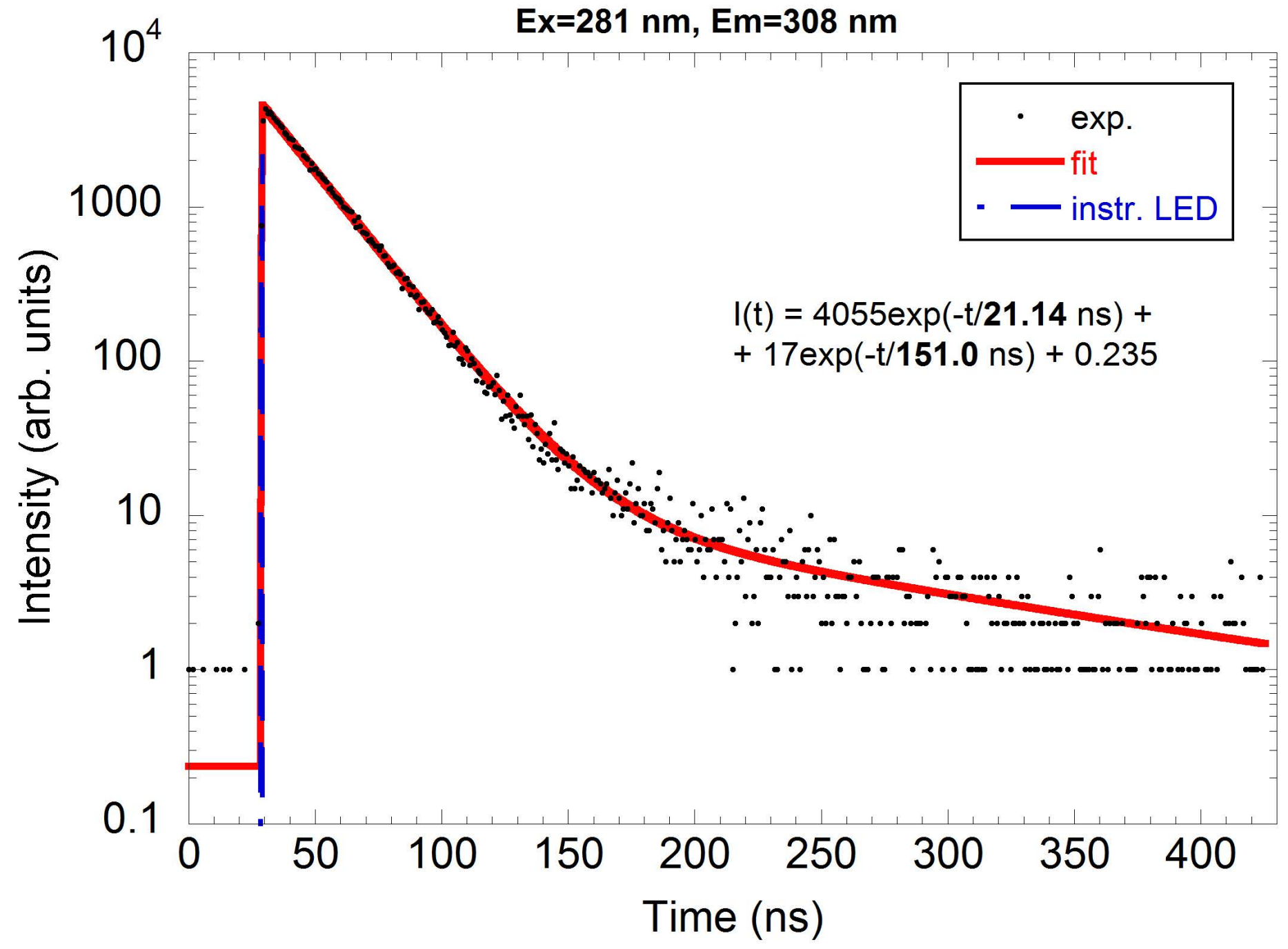


Fig 5

LuAG:Pr $0.2 \%$, Mg 3000 ppm

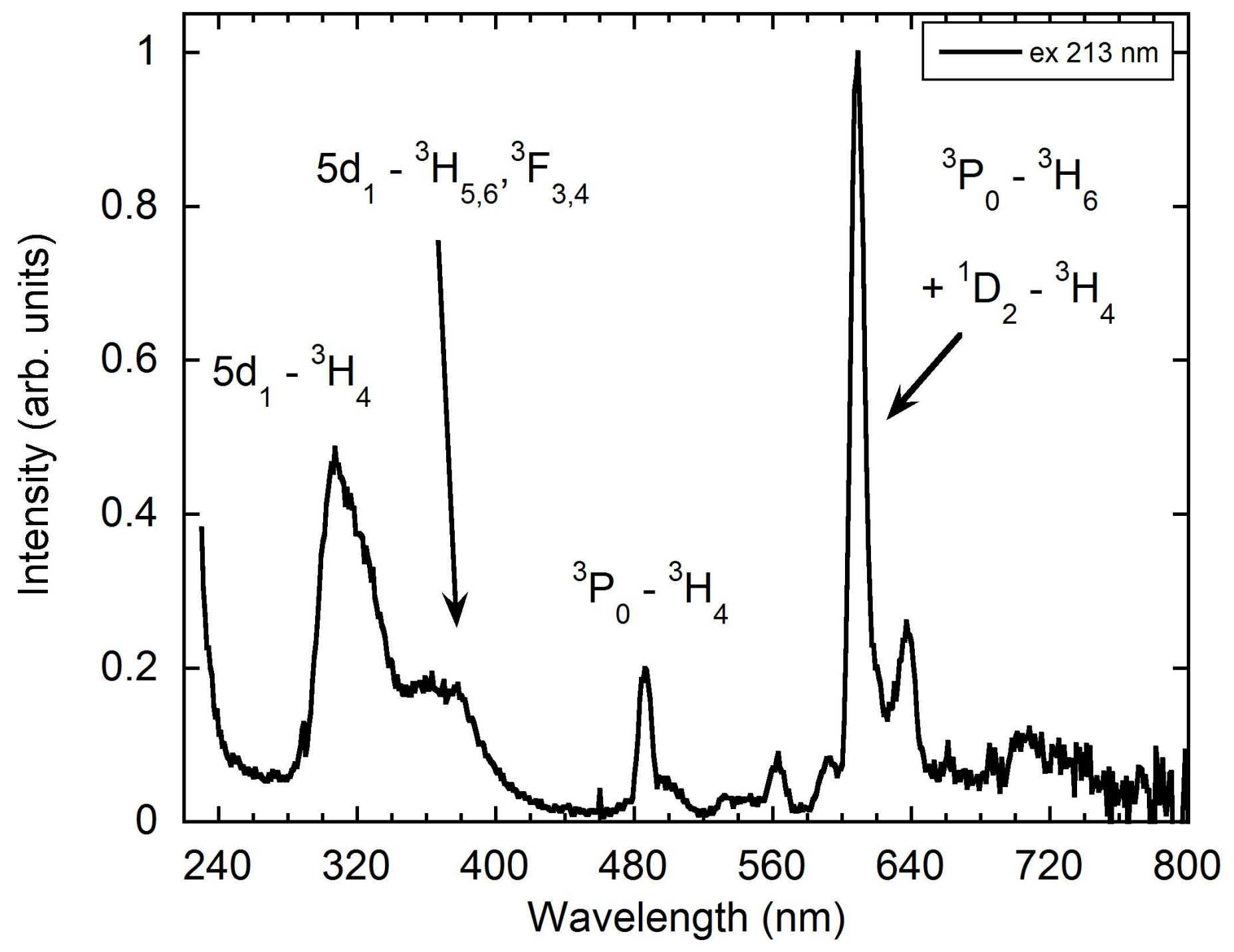


Fig 6

\section{LuAG-Pro.2\% END ${ }^{137} \mathrm{Cs}$}

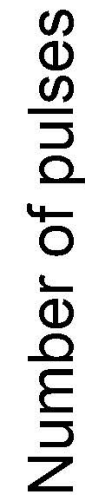

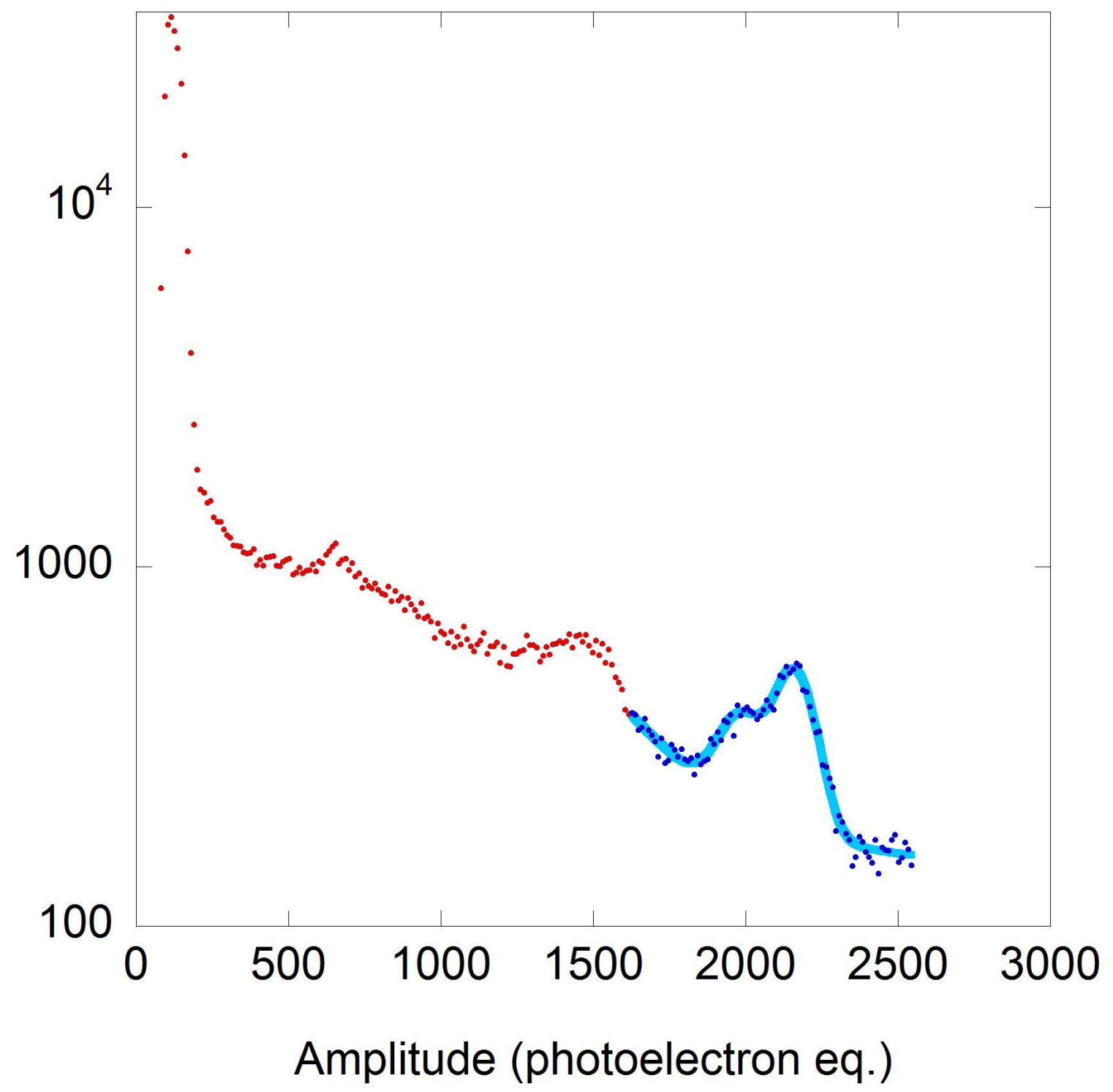


Fig $7 \quad 3500$

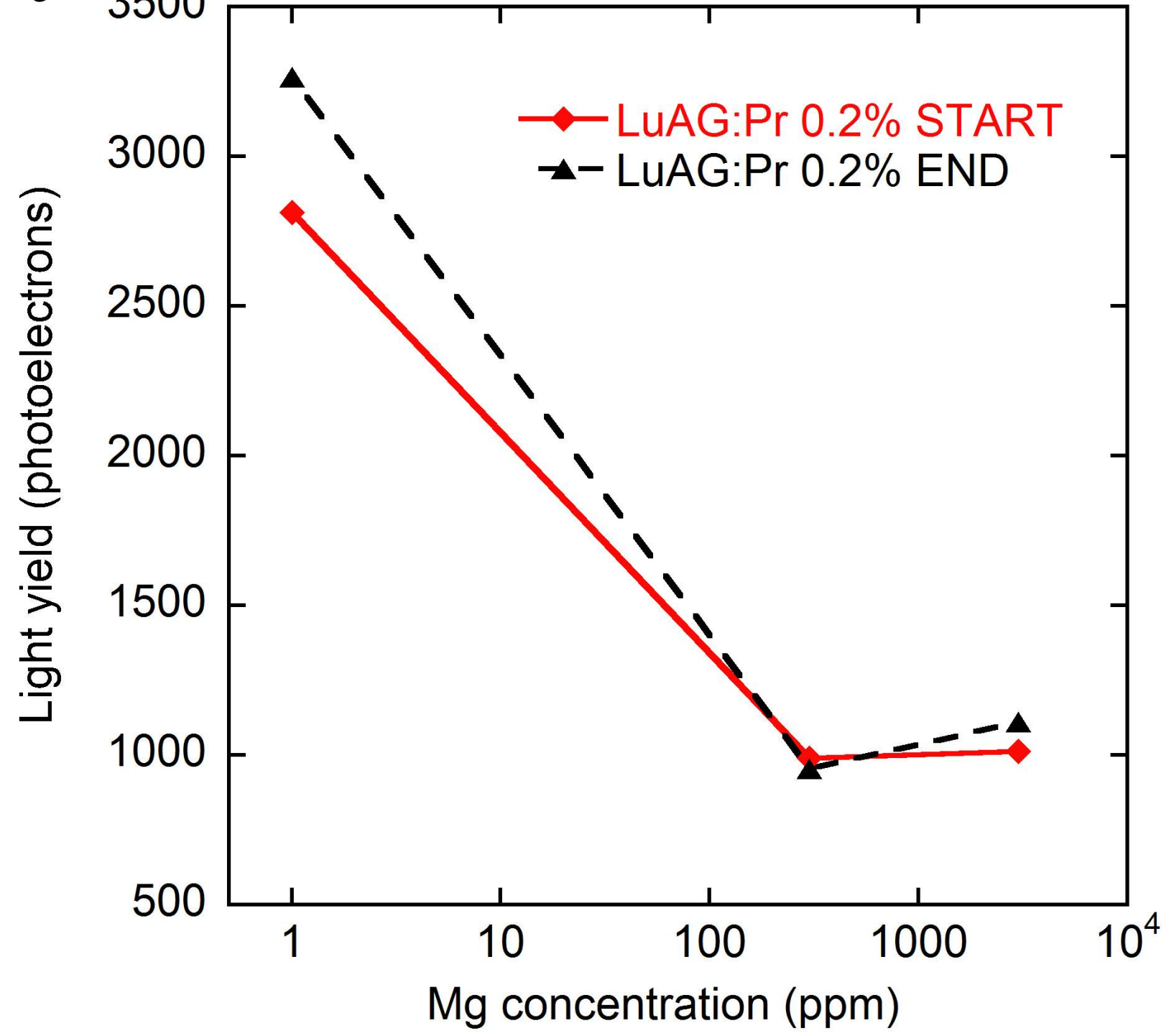


Fig 8a

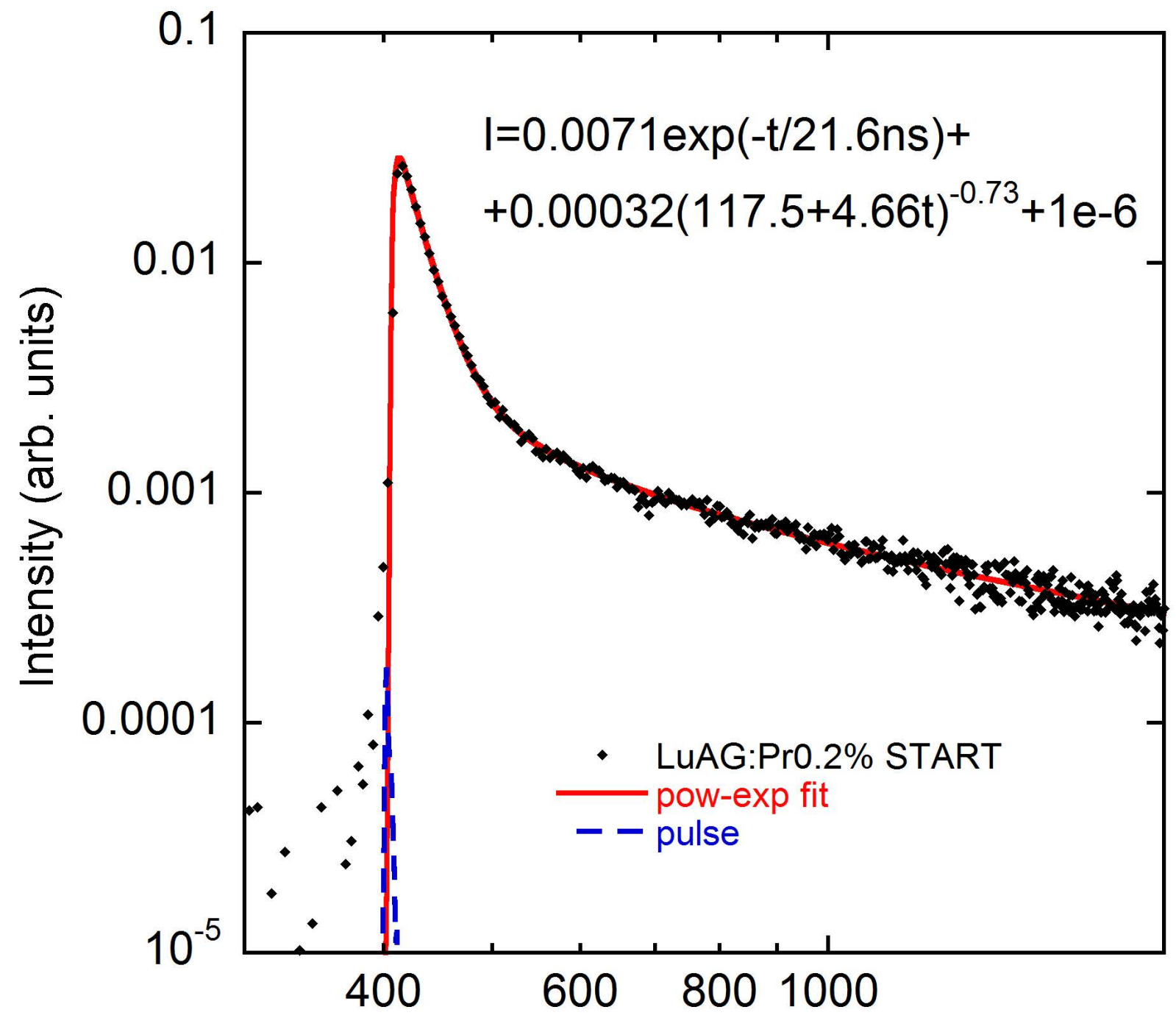

Time (ns) 


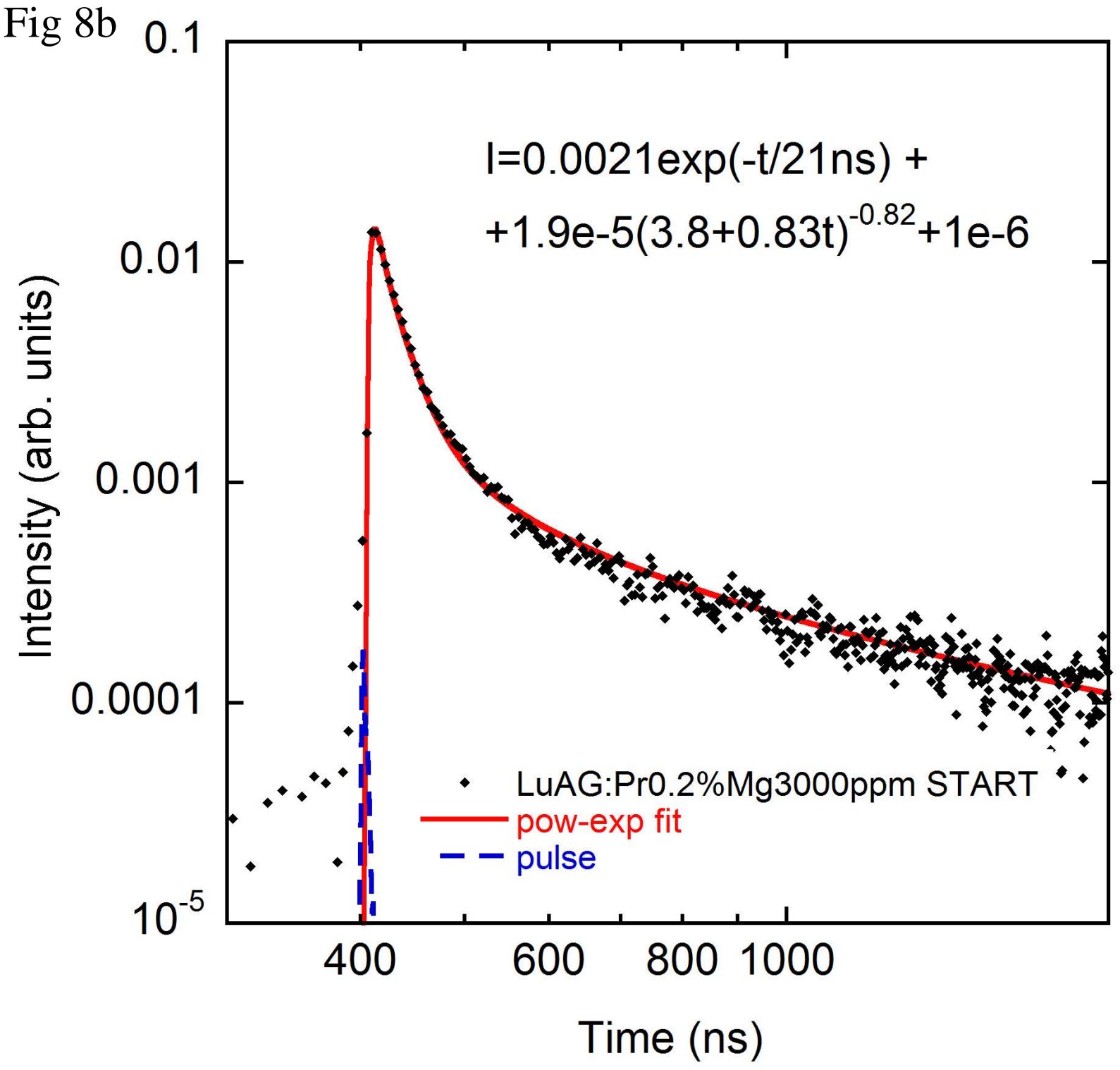


Fig 9

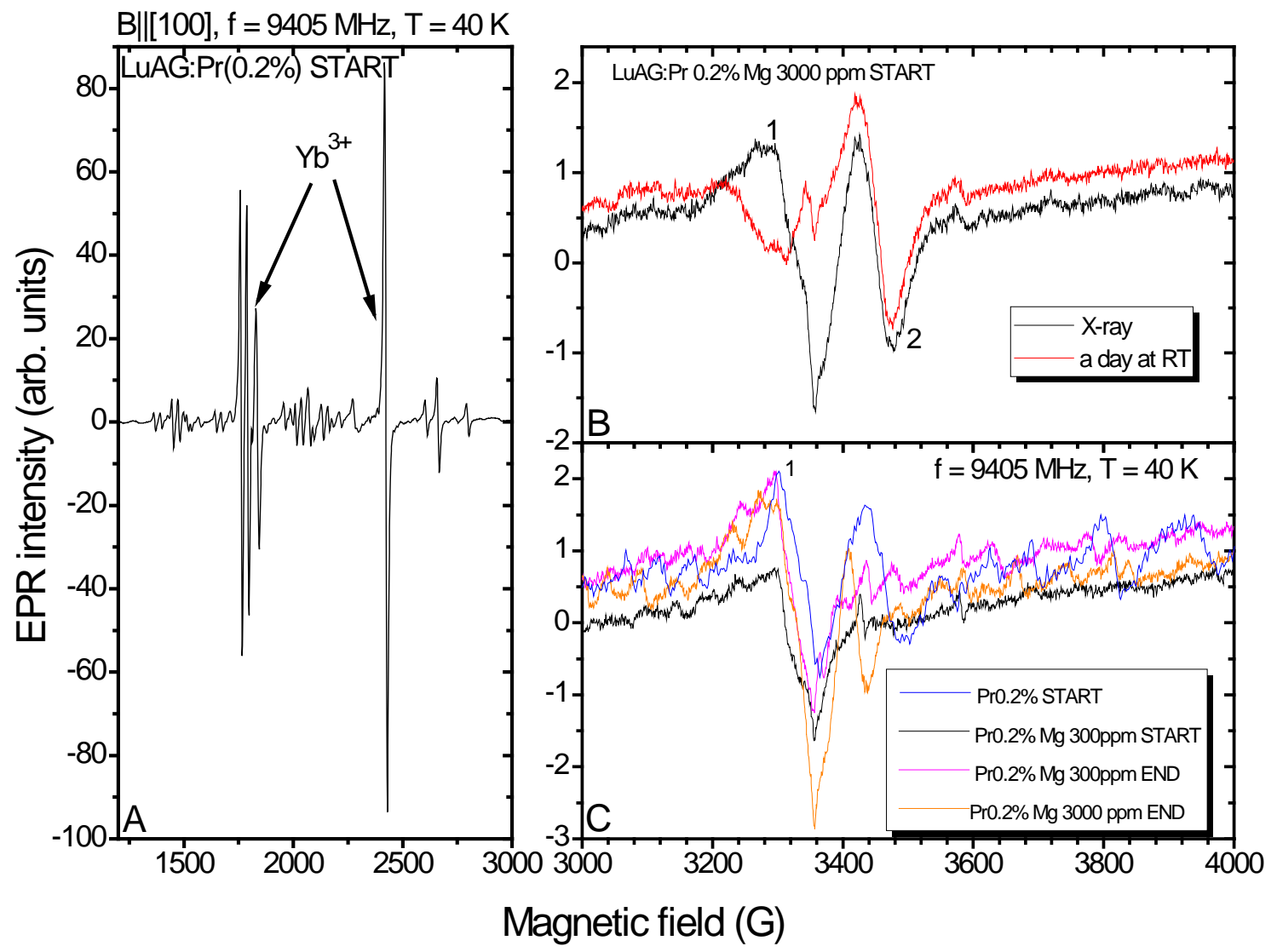


Fig 10

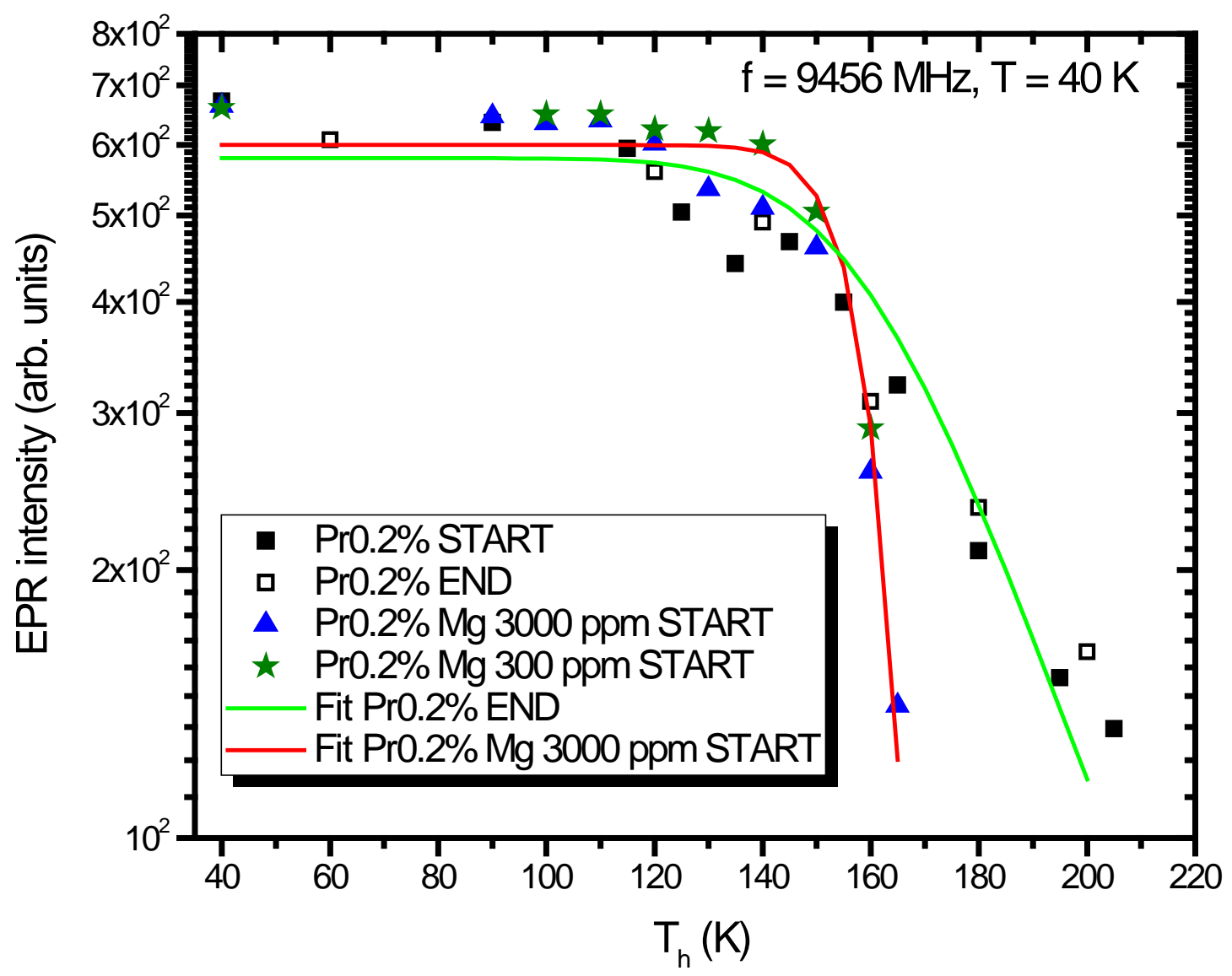

